\title{
Statistical Analysis of Point Defects in Hydrogen Storage Materials
}

\author{
M.C. Sarahan*, Q.M. Ramasse**, D.G. Morgan***, N.D. Browning*,**** \\ * Department of Chemical Engineering and Materials Science, University of California, Davis, \\ One Shields Avenue, Davis, CA 95616 \\ ** National Center for Electron Microscopy, Lawrence Berkeley National Laboratory, MS 72- \\ 150, Berkeley, CA 94720 \\ *** Department of Chemistry, Indiana University, 800 E. Kirkwood Ave., Bloomington, IN \\ 47405 \\ **** Physical and Life Sciences Directorate, Lawrence Livermore National Laboratory, 7000 \\ East Avenue, Livermore, CA 94550
}

Software for rapid multivariate statistical analysis of high-resolution scanning transmission electron microscope images has been developed. The software processes images based on repeat units observed, and provides information visually about the local variation in the repeat unit due to point defects, as well as . This software was used to study the site occupancy of a silicon clathrate material, which has been proposed as a hydrogen storage material [1].

The image processing involved in this analysis includes correction for long-range brightness changes, such as those observed due to thickness variation. From there, a template image representative of the area of interest is obtained. Arbitrary repeat units can be targeted by cropping the original image. As an alternative, facilities for calculating the average unit cell in an image are implemented in a user-friendly graphical interface. With a template defined, all matches for that template are located in the original image, and small sub-images are automatically cropped. Multivariate statistical analysis is then performed on the sub-images to establish ways that the sub-images differ from one another. Output from this analysis is presented to the user visually, with a pseudocolor version of the original image that maps eigenvector values to colors. This output can be used to choose regions of interest for further analysis, such as energy loss spectroscopy. Processing time is less than one minute, so this tool can be applied at the microscope during data acquisition, rather than after a session.

The software was applied to several images of sodium or potassium silicon clathrates with varying alkali cation occupancy. It is proposed that hydrogen can occupy sodium or potassium sites that are vacant. Previous work has demonstrated software to calculate the average unit cell, and thus the average occupancy [2]. The current work takes this further, showing site occupancy at each unit cell, and thus provides spatially resolved site occupation data in an easily interpretable form.

\section{References}

[1] D. Neiner et al. J. Am. Chem. Soc. 129, (2007) 13857-13862

[2] D.G. Morgan, Q.M. Ramasse, N.D. Browning, J. Electron Microsc., in press

[3] This work was supported by the United States Department of Energy under grant number DEFG02-03ER46057. 


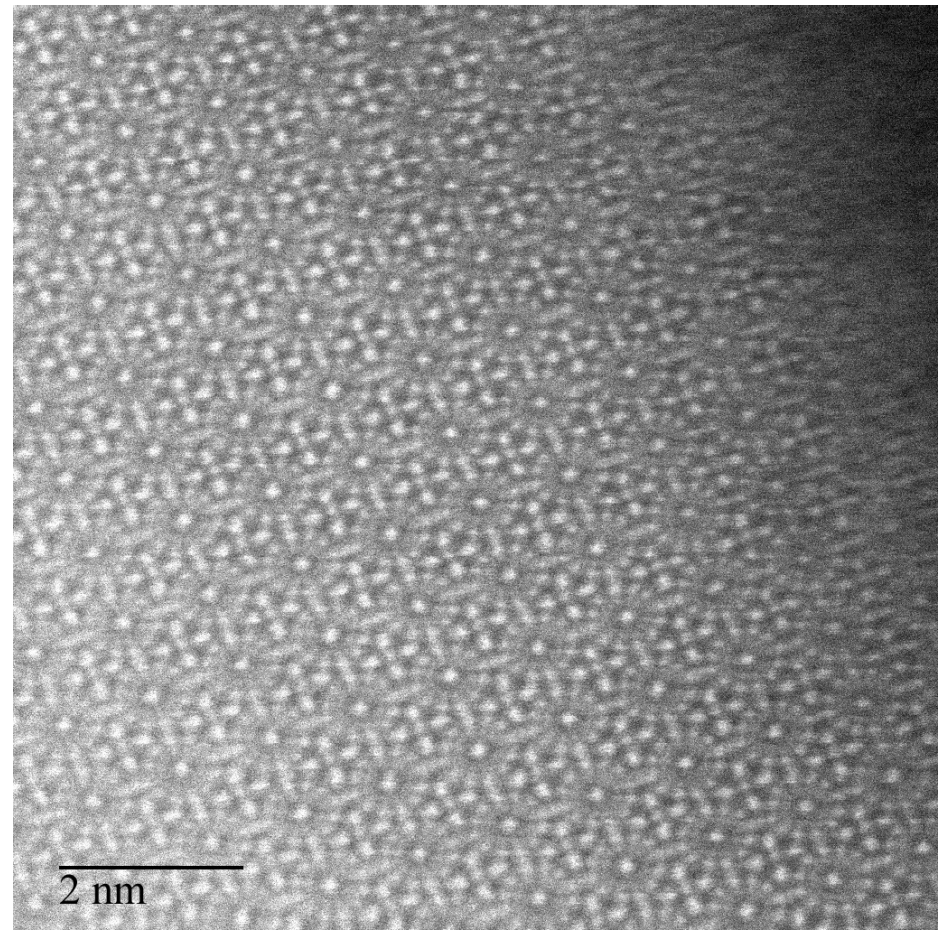

Fig. 1. HAADF-STEM $\{001\}$ zone-axis view of $\mathrm{K}_{\mathrm{x}} \mathrm{Si}_{46}$, a proposed hydrogen storage material.
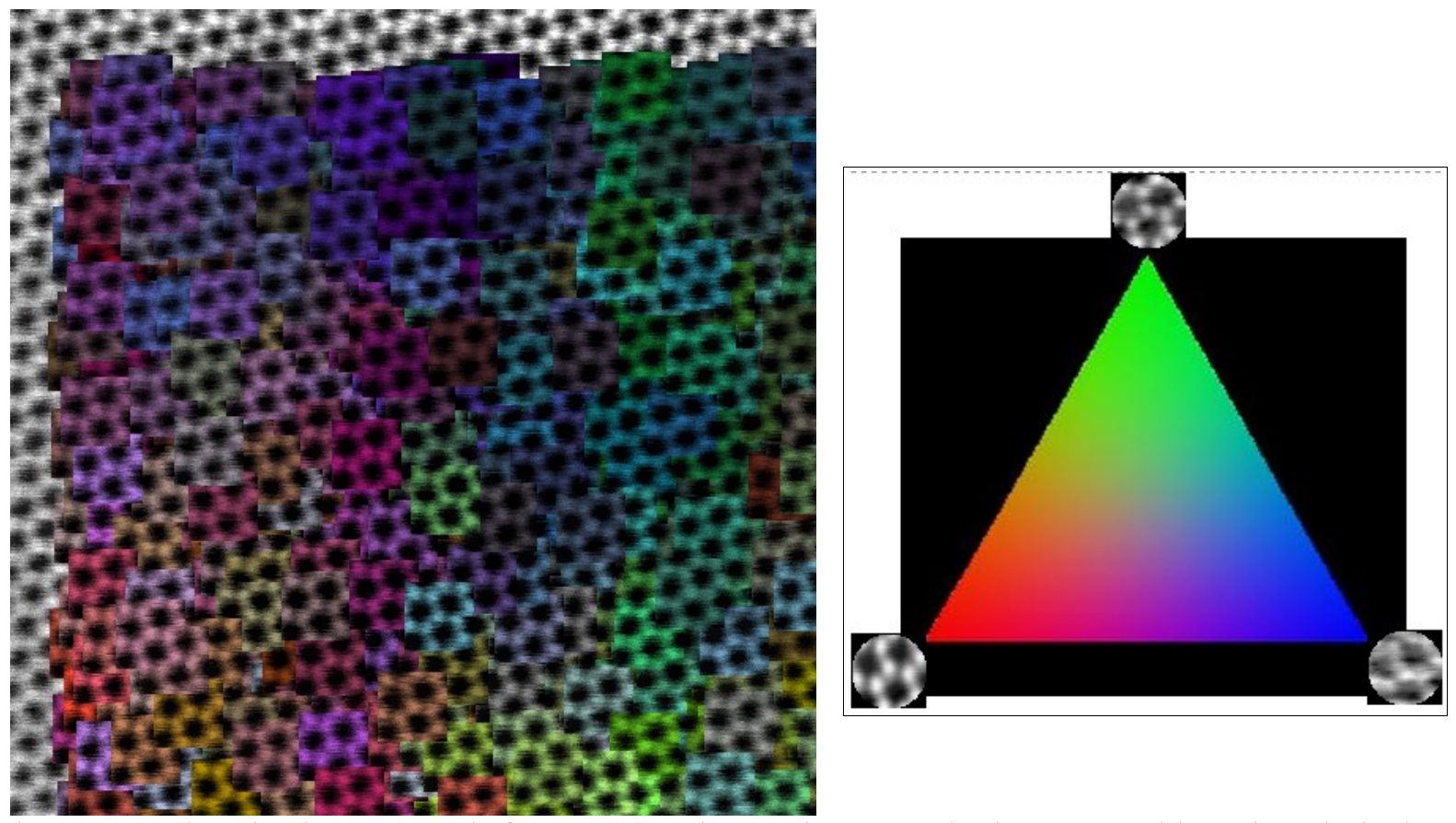

Fig. 2. Pseudocolor image result from processing an image. The image used here is cobalt-doped zinc oxide. This image was used to develop and test the software for hexagonal lattices. The key shows the three eigenvectors that color is mapped to. Pure colors are indicative of one strongly additive eigenvector, and two subtractive eigenvectors, rather than null-contributing eigenvectors. Eigenvectors scale from negative to positive contributions, and this scale is remapped to the $0-255$ range of the $\mathrm{R}, \mathrm{G}, \mathrm{B}$ color system. 